Bull. Korean Math. Soc. 46 (2009), No. 5, pp. 931-940

DOI 10.4134/BKMS.2009.46.5.931

\title{
FOLIATIONS ASSOCIATED WITH PFAFFIAN SYSTEMS
}

\author{
ChONG-KyU HAN
}

\begin{abstract}
Given a system of smooth 1 -forms $\theta=\left(\theta^{1}, \ldots, \theta^{s}\right)$ on a smooth manifold $M^{m}$, we give a necessary and sufficient condition for $M$ to be foliated by integral manifolds of dimension $n, n \leq p:=m-s$, and construct an integrable supersystem $(\theta, \eta)$ by finding additional 1-forms $\eta=\left(\eta^{1}, \ldots, \eta^{p-n}\right)$. We also give a necessary and sufficient condition for $M$ to be foliated by reduced submanifolds of dimension $n, n \geq p$, and construct an integrable subsystem $\left(d \rho^{1}, \ldots, d \rho^{m-n}\right)$ by finding a system of first integrals $\rho=\left(\rho^{1}, \ldots, \rho^{m-n}\right)$. The special case $n=p$ is the Frobenius theorem on involutivity.
\end{abstract}

\section{Introduction and preliminaries}

Let $M$ be a germ of a smooth $\left(C^{\infty}\right)$ manifold of dimension $m$. By a Pfaffian system we mean a system

$$
\theta:=\left(\theta^{1}, \ldots, \theta^{s}\right)
$$

of smooth 1-forms that are linearly independent. A dual expression of (1) is the subbundle $\mathcal{D}$ of $T M$ consisting of the tangent vectors $(x, V) \in T M$ that is annihilated by $\theta$, namely, at each point $x \in M$

$$
\mathcal{D}_{x}=\left\{V \in T_{x} M:\left\langle\theta^{\alpha}, V\right\rangle=0, \quad \alpha=1, \ldots, s\right\} .
$$

$\mathcal{D}$ is a subbundle because $\theta^{\alpha}$ are independent. Let $p:=m-s$. Associated with the Pfaffian system (1) there are two types of submanifolds of basic importance:

Definition 1. A smooth submanifold $N$ of dimension $n, n \leq p$, is called an integral manifold of (1) if one of the following equivalent conditions holds:

i) $T_{x} N \subset \mathcal{D}_{x}, \quad \forall x \in N$.

ii) For the inclusion map $i: N^{n} \hookrightarrow M^{m}$ we have

$$
i^{*} \theta^{\alpha}=0, \quad \alpha=1, \ldots, s .
$$

Received July 31, 2008 .

2000 Mathematics Subject Classification. 35N10, 58A15, 32F25.

Key words and phrases. Pfaffian system, integral manifolds, reduced manifolds, foliation, Frobenius integrability.

The author was partially supported by KRF 2005-070-C00007 of Korea Research Foundation. 
Definition 2. A smooth submanifold $N$ of dimension $n, n \geq p$, is called a reduced submanifold of (1) if and only if one of the following equivalent conditions holds:

i) $T_{x} N \supset \mathcal{D}_{x}, \quad \forall x \in N$.

ii) $\left\{i^{*} \theta^{1}, \ldots, i^{*} \theta^{s}\right\}$ has rank $n-p$.

The equivalence of i) and ii) in Definition 2 is shown in Theorem 2.1. Observe that when $n=p$ these two notions are identical. The Pfaffian system (1) is said to be integrable (or involutive) if

$$
d \theta^{\alpha}=0, \quad \bmod \theta, \quad \alpha=1, \ldots, s . \quad \text { (integrability conditions) }
$$

We state the Frobenius theorem as follows:

Theorem 3. Suppose that the Pfaffian system (1) satisfies the integrability conditions (3). Then for any point $x \in M$ there exists a uniquely determined integral manifold $N$ of dimension $p:=m-s$ through $x$. Thus $M$ is foliated by a s-parameter family of integral manifolds of dimension $p$. Conversely, if $M$ is foliated by integral manifolds of dimension $p$ then the Pfaffian system $\theta$ satisfies (3).

The standard proof as in [15] of Theorem 3 is based on the fundamental theorem of ordinary differential equations. The latter, in turn, is based on the convergence of the successive approximation of the integral operator that solves the initial value problem for the ordinary differential equation, see [4]. In this paper we shall study foliation by integral manifolds of dimension $n, n \leq p$, and by reduced submanifolds of dimension $n, n \geq p$. The Frobenius theorem is the special case $n=p$, where these two foliations coincide. Now let $\left\{\omega^{1}, \ldots, \omega^{p}\right\}$ be a set of closed 1 -forms that completes $\theta$ to a coframe $\left\{\theta^{1}, \ldots, \theta^{s}, \omega^{1}, \ldots, \omega^{p}\right\}$ over $M$. We set for each $\alpha=1, \ldots, s$,

$$
d \theta^{\alpha}=\sum_{\substack{i<j \\ i, j=1, \ldots, p}} T_{i j}^{\alpha} \omega^{i} \wedge \omega^{j}, \quad \bmod \theta .
$$

Arranging the pairs $(i j)$ with $i<j$ in lexicographical order, we array the coefficients in $s \times\left(\begin{array}{l}p \\ 2\end{array}\right)$ matrix

$$
\mathcal{T}=\left[T_{i j}^{\alpha}\right]
$$

We shall call $\mathcal{T}$ the torsion matrix and $\sum_{i<j} T_{i j}^{\alpha} \omega^{i} \wedge \omega^{j}$ the torsion tensor for (1). Theorem 3 is the extreme case that $\mathcal{T}$ has rank zero, that is, all $T_{i j}^{\alpha}$ are identically zero. By checking the rank and by analyzing the linear dependence of columns (or rows) of $\mathcal{T}$ we obtain various generalizations of Theorem 3 . In [10] the author presented conditions on $\mathcal{T}$ for there to exist $s^{\prime}$-parameter, $\left(s^{\prime}<s\right)$, family of integral manifolds of dimension $p$ and the conditions that imply the existence of a single integral manifold of dimension $p^{\prime}, p^{\prime} \leq p$. [11] and [12] are the applications. In the present paper we clarify the conditions for $M$ to be foliated by integral manifolds of dimension $n, n \leq p$ (Theorem 1.1). 
Now we recall the classical notion of the first integral (constant of motion). For a smooth nonvanishing vector field $X$ on $M^{m}$ the first integral is a smooth real-valued non-constant function $u$ that satisfies the first order linear partial differential equation $X u=0$. Locally, there exist $m-1$ functionally independent first integrals. However, for a system of vector fields $X_{1}, \ldots, X_{p}, p \geq 2$, generically there are no first integrals, that is, if $X_{j} u=0$ for all $j=1, \ldots, p$, then $u$ is constant. In $1840 \mathrm{R}$. Deahna [5] discovered that if the commutators $\left[X_{i}, X_{j}\right]$ is in the linear span of $X^{\prime} s$, then there exists maximal number $(m-p)$ of first integrals. Later in 1866, A. Clebsch [3] first gave a complete proof of this theorem and in $1877 \mathrm{G}$. Frobenius [6] first used $d$ expressing the theorem in terms of differential forms $\theta$ and their exterior derivatives $d \theta$, as we stated in Theorem 3. The notion of the first integral of (1) is naturally defined in the theory of derived flag as in [2, Chapter 1]. We present the same ideas in Theorem 2.2 in elementary and algorithmic way so that one can construct the first integral explicitly. If $\rho=\left(\rho^{1}, \ldots, \rho^{\sigma}\right), \sigma \leq s$, with $d \rho^{1} \wedge \cdots \wedge d \rho^{\sigma} \neq 0$, is a system of first integrals, then each level set of $\rho$ is a reduced submanifold so that $M$ is foliated by $\sigma$-parameter family of reduced submanifolds of dimension $m-\sigma$. The case $\sigma=s$ is that of the Frobenius theorem.

Now we recall some basic definitions and notations: By $C^{\infty}(M)$ we denote the ring of smooth real-valued functions on $M$. For each $k=1, \ldots, m$, let $\Omega^{k}$ be the module over $C^{\infty}(M)$ of all smooth differential $k$-forms. Let $\Omega^{0}=C^{\infty}(M)$, the 0 -forms. We denote by $\Omega^{*}:=\bigoplus_{k=0}^{m} \Omega^{k}$ the exterior algebra of smooth differential forms on $M$. A subalgebra $\mathcal{I}$ is called an algebraic ideal if

i) $\mathcal{I} \wedge \Omega \subset \mathcal{I}$

ii) $\phi=\sum_{k=0}^{m} \phi_{k} \in \mathcal{I}$, where $\phi_{k} \in \Omega^{k}$, then each component $\phi_{k} \in \mathcal{I}$ (homogeneity condition).

We easily see that an algebraic ideal is two-sided by the homogeneity condition. Let $\mathcal{I}$ be an algebraic ideal. For $\phi, \eta \in \Omega^{*}$ we write

$$
\phi \equiv \eta \bmod \mathcal{I}
$$

if $\phi-\eta \in \mathcal{I}$.

In this paper we consider only algebraic ideals generated by finitely many 0 -forms and 1-forms: Let $\mathcal{I}$ be an algebraic ideal of $\Omega^{*}$ generated by a finite set of 0 -forms $\rho=\left(\rho^{1}, \ldots, \rho^{d}\right)$ and a finite set of 1 -forms $\theta=\left(\theta^{1}, \ldots, \theta^{s}\right)$. Then $\mathcal{I}$ is the set of all elements of $\Omega^{*}$ of the form $\sum_{j=1}^{d} \rho^{j} \phi_{j}+\sum_{\alpha=1}^{s} \theta^{\alpha} \wedge \psi_{\alpha}$, for $\phi_{j}, \psi_{\alpha} \in \Omega^{*}$. We denote by $\mathcal{I}(\rho, \theta)$ or simply by $(\rho, \theta)$ the algebraic ideal generated by the system of 0 -forms $\rho$ and the system of 1 -forms $\theta$. If $\phi-\eta$ belongs to the ideal $(\rho, \theta)$ we write

$$
\phi \equiv \eta \quad \bmod (\rho, \theta) .
$$

A system of smooth real-valued functions $\rho=\left(\rho^{1}, \ldots, \rho^{d}\right)$ is said to be nondegenerate if

$$
d \rho^{1} \wedge \cdots \wedge d \rho^{d} \neq 0, \quad \bmod \left(\rho^{1}, \ldots, \rho^{d}\right) .
$$


Then a level sets $\rho^{-1}(c)$, for each $c$ in an open subset of $\mathbb{R}^{d}$, are smooth submanifolds of $M$ of dimension $m-d$.

Finally, we mention the following observation: Let $N^{n}$ be a submanifold of $M^{m}$ and let $\mathcal{V} \subset T M$ be a vector bundle of rank $p$ over $N$. Then there exists a set of non-degenerate real-valued functions $\rho=\left(\rho^{1}, \ldots, \rho^{d}\right)$, where $d=m-n$, and a system of independent 1-forms $\theta=\left(\theta^{1}, \ldots, \theta^{s}\right)$, where $s=$ $m-p$, such that $(x, V) \in \mathcal{V}$ if and only if $(x, \mathcal{V})$ is annihilated by $(\rho, \theta)$. There is one-to-one correspondence between the set of such vector bundles and the set of ideals generated by nondegenerate system of functions and independent 1-forms. Some of the ideas and results of this paper are in the classical theory of exterior differential systems. In particular, foliation by lower dimensional $(n<p)$ integral manifolds in the Examples 1.2, 1.3, and 1.4 follows from the Darboux theorem on the rank of a 1 -form $\theta$ ([2, page 40]), which is the case $s=1$ in (1). Our method is constructive and applicable to more general cases.

\section{$\S 1$. Foliation by integral manifolds}

Given a Pfaffian system $\theta=\left(\theta^{1}, \ldots, \theta^{s}\right)$ on $M^{m}$ as in (1) we study in this section the conditions that $M$ is foliated by integral manifolds of dimension $n$, $n \leq m-s$. This is the problem of finding the smallest integrable supersystem of (1). Let $\omega^{1}, \ldots, \omega^{p}, s+p=m$, be closed 1-forms that completes $\theta$ to a coframe

$$
\left\{\theta^{1}, \ldots, \theta^{s}, \omega^{1}, \ldots, \omega^{p}\right\} .
$$

Let $\mathcal{D}$ be the subbundle of $T(M)$ consisting of the tangent vectors that are annihilated by $\theta$. Now coming back to the Pfaffian system (1) we notice first that the case $n=1$ is a problem of ODE: From the fundamental theorem of ODE it follows that $M$ admits foliation by integral curves. Suppose $M$ is foliated by integral manifolds of dimension $n, 2 \leq n \leq p-1$. We assume that on each leaf of the foliation

$$
\omega^{1} \wedge \cdots \wedge \omega^{n} \neq 0
$$

We use the summation convention with the following ranges of indices:

$$
\begin{aligned}
& 1 \leq i, j \leq p, \\
& 1 \leq k, \ell \leq n, \\
& 1 \leq \mu, \nu \leq d:=p-n, \\
& 1 \leq \alpha \leq s .
\end{aligned}
$$

By (1.1), for each $\nu$ there exist smooth functions $a_{\ell}^{\nu}$ such that

$$
\omega^{n+\nu}=a_{\ell}^{\nu} \omega^{\ell} .
$$

Let $\eta=\left(\eta^{1}, \ldots, \eta^{d}\right)$ where

$$
\eta^{\nu}:=\omega^{n+\nu}-a_{\ell}^{\nu} \omega^{\ell} \quad \text { for } \quad \nu=1, \ldots, d .
$$


Now substituting (1.3) in (4) for $\omega^{n+\nu}$ we have

(1.4)

$$
\begin{aligned}
d \theta^{\alpha}= & \sum_{k<\ell}\left\{T_{k \ell}^{\alpha}+T_{k, n+\nu}^{\alpha} a_{\ell}^{\nu}-T_{\ell, n+\nu}^{\alpha} a_{k}^{\nu}+\sum_{\mu<\nu} T_{n+\mu, n+\nu}^{\alpha}\left(a_{k}^{\mu} a_{\ell}^{\nu}-a_{\ell}^{\mu} a_{k}^{\nu}\right)\right\} \omega^{k} \wedge \omega^{\ell}, \\
& \bmod (\theta, \eta)
\end{aligned}
$$

for all $\alpha=1, \ldots, s$.

Then by Theorem 3 the Pfaffian system $(\theta, \eta)$ is integrable, namely,

$$
\begin{aligned}
& d \theta^{a}=0, \quad \bmod (\theta, \eta), \quad \alpha=1, \ldots, s, \\
& d \eta^{\nu}=0, \quad \bmod (\theta, \eta), \quad \nu=1, \ldots, d .
\end{aligned}
$$

Then by (1.3), (1.4) and (1.5) we have

$$
T_{k \ell}^{\alpha}+T_{k, n+\nu}^{\alpha} a_{\ell}^{\nu}-T_{\ell, n+\nu}^{\alpha} a_{k}^{\nu}+\sum_{\mu<\nu} T_{n+\mu, n+\nu}^{\alpha}\left(a_{k}^{\mu} a_{\ell}^{\nu}-a_{\ell}^{\mu} a_{k}^{\nu}\right)=0
$$

for $\alpha=1, \ldots, s$ and for pairs $(k, \ell)$ with $k<\ell$ and $k, \ell=1, \ldots, n$. Now for any smooth function $f$ let $f, \alpha$ and $f,{ }_{j}$ be the smooth functions that are uniquely determined by

Then we have from (1.3)

$$
d f=f,{ }_{\alpha} \theta^{\alpha}+f,{ }_{j} \omega^{j} .
$$

$$
\begin{aligned}
d \eta^{\nu} & =-d a_{\ell}^{\nu} \wedge \omega^{\ell} \\
& =-\left(a_{\ell, k}^{\nu} \omega^{k}+a_{\ell, n+\mu}^{\nu} \omega^{n+\mu}\right) \wedge \omega^{\ell}, \quad \bmod \theta \\
& =-\left(a_{\ell, k}^{\nu}+a_{\ell, n+\mu}^{\nu} a_{k}^{\mu}\right) \omega^{k} \wedge \omega^{\ell}, \quad \bmod (\theta, \eta) \\
& =\sum_{k<\ell}\left(a_{k, \ell}^{\nu}+a_{k, n+\mu}^{\nu} a_{\ell}^{\mu}-a_{\ell, k}^{\nu}-a_{\ell, n+\mu}^{\nu} a_{k}^{\mu}\right) \omega^{k} \wedge \omega^{\ell}, \quad \bmod (\theta, \eta) .
\end{aligned}
$$

From (1.5) and (1.7) it follows that

$$
a_{k, \ell}^{\nu}+a_{k, n+\mu}^{\nu} a_{\ell}^{\mu}-a_{\ell, k}^{\nu}-a_{\ell, n+\mu}^{\nu} a_{k}^{\mu}=0
$$

for each pair $(k, \ell)$ with $k<\ell, k, \ell=1, \ldots, n$. Thus we proved the following:

Theorem 1.1. Suppose $\theta=\left(\theta^{1}, \ldots, \theta^{s}\right)$ is a system of smooth 1-forms that are defined and linearly independent on a smooth manifold $M$ of dimension $m$. Let $p=m-s$ and $n$ be an integer with $2 \leq n \leq p$. Then $M$ is foliated by integral manifolds of dimension $n$ if and only if there exists a system of 1 forms $\eta=\left(\eta^{1}, \ldots, \eta^{d}\right)$, where $d=p-n$, so that $(\theta, \eta)$ satisfies the integrability conditions. Such $\eta$ is given by a system of smooth functions $a_{k}^{\nu}, \nu=1, \ldots, d$, $k=1, \ldots, n$, that satisfies (1.6) and (1.8).

We call $\eta$ complementary 1-forms. In (1.6) and (1.8) the number of unknown functions $a_{k}^{\mu}$ is $d n$. (1.6) is a system of $s\left(\begin{array}{c}n \\ 2\end{array}\right)$ quadratic equations and (1.8) is a system of $d\left(\begin{array}{c}n \\ 2\end{array}\right)$ partial differential equations of first order, respectively, for the unknown functions $a_{k}^{\mu}$. Let us consider the following simplest cases:

Case 1: Single 1-form on $M^{4}: s=1, p=3, n=2, d=1$. 
Case 2: Single 1-form on $M^{5}: s=1, p=4, n=3, d=1$.

Case 3: Single 1-form on $M^{5}: s=1, p=4, n=2, d=2$.

Case 4: Two 1-forms on $M^{5}: s=2, p=3, n=2, d=1$.

In each of these cases, we compare the number of unknown functions and number of equations as follows:

$\left.\begin{array}{cccc}\text { case } & \text { unknowns } & \text { equations }(1.6) & \text { equations }(1.8) \\ & d n & s\left(\begin{array}{l}n \\ 2\end{array}\right) & d\left(\begin{array}{c}n \\ 2\end{array}\right)\end{array}\right)$

Comparing the number of unknowns and that of equations we see that the case 1) is determined and 3) is underdetermined. Cases 2) and 4) are overdetermined. In determined and underdetermined cases, it is easy to construct complementary set of 1 -forms $\eta$ that completes $\theta$ to an involutive system $(\theta, \eta)$.

Example 1.2. Case 1 that admits foliation by integral manifolds of dimension 2: Let $M=\mathbb{R}^{4}$ and $\theta=d x^{4}+x^{2} d x^{3}$. Then $\eta:=d x^{3}+g\left(x^{2}, x^{3}, x^{4}\right) d x^{2}$, where $g$ is arbitrary, is the complementary 1-form that we look for.

Naturally it gives rise to the following questions: In determined and underdetermined cases are (1.6) and (1.8) always solvable? If not, in what dimensions are they solvable? To be easiest, consider the cases $M^{m}, s=1, n=2$. Then $d=m-3$, the number of unknowns is $2(m-3)$, the number of equations is $1+(m-3)$, therefore, underdetermined if $m \geq 5$. Then the question is whether (1.6) and (1.8) are solvable for sufficiently large $m$.

Example 1.3. Case 2 that has foliation by integral manifolds of dimension 3 : Let $M=\mathbb{R}^{5}$. Given $\theta=x^{3} d x^{4}+d x^{5}, \eta:=d x^{4}+h\left(x^{3}, x^{4}, x^{5}\right) d x^{3}$ is a complementary 1 -form that gives an involutive system $(\theta, \eta)$.

Example 1.4. Case 2 that admits no foliation by integral 3-manifolds: In $\mathbb{R}^{5}$ let $\theta=x^{1} d x^{2}+x^{3} d x^{4}+d x^{5}$. Suppose $\eta=\sum_{j=1}^{4} a_{j} d x^{j}$, $\bmod \theta$, is a complementary 1-form. We normalize the last coefficient to be 1 , so that

$$
\eta=d x^{4}-\left(f d x^{1}+g d x^{2}+h d x^{3}\right), \bmod \theta .
$$

Then we have

$$
\begin{aligned}
d \theta & =d x^{1} \wedge d x^{2}+d x^{3} \wedge d x^{4} \\
& =d x^{1} \wedge d x^{2}-f d x^{1} \wedge d x^{3}-g d x^{2} \wedge d x^{3}, \bmod (\theta, \eta)
\end{aligned}
$$

which cannot be zero. Other way of normalization leads to the same situation. Therefore, there is no complementary 1-form $\eta$. 
Example 1.5. Case 4 that has foliation by integral 2 -manifolds: In $\mathbb{R}^{5}$ let

$$
\begin{aligned}
& \theta^{1}=\left(x^{1}+x^{3}\right) d x^{2}+d x^{5}, \\
& \theta^{2}=\left(x^{2}+x^{3}\right) d x^{1}+d x^{4} .
\end{aligned}
$$

We look for foliation with $d x^{1} \wedge d x^{2} \neq 0$. Let us find a complementary 1-form

$$
\eta=d x^{3}-\left(f d x^{1}+g d x^{2}\right) .
$$

Since

$$
\begin{aligned}
& d \theta^{1}=d x^{1} \wedge d x^{2}+d x^{2} \wedge d x^{3}=(1+f) d x^{1} \wedge d x^{2}, \quad \bmod (\theta, \eta), \\
& d \theta^{2}=-d x^{1} \wedge d x^{2}-d x^{1} \wedge d x^{3}=-(1+g) d x^{1} \wedge d x^{2}, \bmod (\theta, \eta),
\end{aligned}
$$

by setting the right side to be zero we have $f=g=-1$, which gives

$$
\eta=d x^{3}+d x^{1}+d x^{2} .
$$

Then $\left(\theta^{1}, \theta^{2}, \eta\right)$ is involutive.

Example 1.6. Case 4 that has no foliation by integral 2 -manifolds: In $\mathbb{R}^{5}$ let

$$
\begin{aligned}
& \theta^{1}=\left(x^{1} x^{2}+x^{3}\right) d x^{2}+d x^{5} \\
& \theta^{2}=\left(2 x^{1} x^{2}+x^{3}\right) d x^{1}+d x^{4}
\end{aligned}
$$

We look for foliation with

$$
d x^{1} \wedge d x^{2} \neq 0
$$

on each leaf. Let $\eta$ as in (1.11) be a complementary 1-form. Then by a similar calculation as in Example 1.5 we have

$$
\begin{aligned}
& d \theta^{1}=x^{2} d x^{1} \wedge d x^{2}-d x^{2} \wedge d x^{3}=\left(x^{2}+f\right) d x^{1} \wedge d x^{2}, \quad \bmod (\theta, \eta), \\
& d \theta^{2}=-2 x^{1} d x^{1} \wedge d x^{2}-d x^{1} \wedge d x^{3}=-\left(2 x^{1}+g\right) d x^{1} \wedge d x^{2}, \quad \bmod (\theta, \eta) .
\end{aligned}
$$

By setting the right side to be zero we have $f=-x^{2}, g=-2 x^{1}$, which gives

$$
\eta=d x^{3}+x^{2} d x^{1}+2 x^{1} d x^{2} .
$$

Then

$$
d \eta=d x^{1} \wedge d x^{2} \neq 0, \quad \bmod (\theta, \eta),
$$

thus there is no complementary 1 -form.

\section{$\S 2$. Foliation by reduced submanifolds}

In this section we investigate the conditions that $M$ is foliated by reduced submanifolds. In general, foliation of $M$ by submanifolds of dimension $n$ is locally given by a non-degenerate set of smooth functions $\rho=\left(\rho^{1}, \ldots, \rho^{m-n}\right)$ whose level sets $\rho^{-1}(c)$, for all $c$ in some open subset of $\mathbb{R}^{m-n}$, are the leaves of the foliation. First, we prove the following: 
Theorem 2.1. Let $\theta=\left(\theta^{1}, \ldots, \theta^{s}\right)$ be a Pfaffian system on $M^{m}$ and $p=m-s$. Suppose that a submanifold $i: N^{n} \hookrightarrow M^{m}, n \geq p$, is defined as a zero locus of a system of nondegenerate functions $\rho=\left(\rho^{1}, \ldots, \rho^{m-n}\right)$, that is, $\rho \circ i=0$. Then the following are equivalent:
i) $N$ is a reduced submanifold for the Pfaffian system $\theta$.
ii) $(\rho, \theta) \supset(\rho, d \rho)$.
iii) $d \rho^{j}=0, \quad \bmod (\rho, \theta), \quad j=1, \ldots, m-n$.
iv) $\left\{i^{*} \theta^{1}, \ldots, i^{*} \theta^{s}\right\}$ has rank $n-p$.

Proof. Since ii) is the dual expression of that $\mathcal{D}_{x} \subset T_{x} N, \forall x \in N$, we have i) $\Leftrightarrow$ ii).
ii) $\Rightarrow$ iii) is obvious
iii) $\Rightarrow$ iv) Set

$$
d \rho^{j}=\sum_{k=1}^{m-n} \rho^{k} \omega_{k}^{j}+\sum_{\alpha=1}^{s} a_{\alpha}^{j} \theta^{\alpha}
$$

for some 1-forms $\omega_{k}^{j}$ and 0-forms $a_{\alpha}^{j}$. Since

$$
d \rho^{1} \wedge \cdots \wedge d \rho^{m-n} \neq 0, \quad \bmod (\rho),
$$

at each point $x \in N$, the matrix

$$
\left(a_{\alpha}^{j}(x)\right) ; j=1, \ldots, m-n, \alpha=1, \ldots, s
$$

has rank $m-n$. Pulling back (2.1) by $i$ we have

$$
0=\sum_{\alpha=1}^{s}\left(a_{\alpha}^{j} \circ i\right)\left(i^{*} \theta^{\alpha}\right) \quad \text { for each } j=1, \ldots, m-n .
$$

Thus $\left\{i^{*} \theta^{\alpha}, \alpha=1, \ldots, s\right\}$ satisfies $m-n$ independent linear equations, therefore, has rank $s-(m-n)=n-(m-s)=n-p$.

iv) $\Rightarrow$ i) Suppose that $\left\{i^{*} \theta^{\alpha}\right\}$ has rank $n-p$. Then this defines a subbundle $\mathcal{D}^{\prime} \subset T N$ of rank $n-(n-p)=p$. Thus for each $x \in N$ both of $\mathcal{D}_{x}^{\prime}=\mathcal{D}_{x} \cap T_{x} N$ and $\mathcal{D}_{x}$ has dimension $p$. Therefore, $\mathcal{D}_{x} \cap T_{x} N=\mathcal{D}_{x}$, which implies $\mathcal{D}_{x} \subset$ $T_{x} N$.

To find the functions $\rho=\left(\rho^{1}, \ldots, \rho^{m-n}\right)$ in Theorem 2.1 we apply $d$ to $(2.1)$ :

$$
0=\sum_{\alpha=1}^{s} a_{\alpha}^{j} d \theta^{\alpha}, \quad \bmod (\rho, \theta) .
$$

Since the matrix $\left(a_{\alpha}^{j}(x)\right), x \in N$, has rank $m-n, d \theta^{\alpha}$ satisfies $m-n$ independent linear equations, therefore, the torsion tensor (4) has rank $s-(m-n)=$ $n-p$. This implies that every square submatrix of size $n-p+1$ of the torsion matrix (5) has determinant zero on $N$. We find nondegenerate factors of those determinants and find $\rho$ so that each determinant belongs to the ideal generated by $\rho$. For instance, if $n=m-1, s \leq\left(\begin{array}{l}p \\ 2\end{array}\right)$ we find the determinants of $s \times s$ submatrices and take a non-degenerate common factor of those determinants 
as a possible candidate of $\rho$ and then check this $\rho$ indeed satisfies any of four equivalent conditions of Theorem 2.1. If $\rho$ satisfies iii) of Theorem 2.1 with right hand side independent of $\rho$, namely, if

$$
d \rho=0, \quad \bmod (\theta),
$$

then $M$ is foliated by reduced submanifolds $\rho=$ constant. Thus we have the following:

Theorem 2.2. Given a Pfaffian system (1), $M$ is foliated by reduced submanifolds of dimension $n,(n \geq p)$, if and only if there exists a non-degenerate system of real-valued functions $\rho=\left(\rho^{1}, \ldots, \rho^{m-n}\right)$ such that

$$
d \rho^{\alpha} \equiv 0, \quad \bmod \theta, \quad \forall \alpha=1, \ldots, m-n .
$$

In cases $s \leq\left(\begin{array}{l}p \\ 2\end{array}\right)$, the determinants of all the square submatrices of (5) of size $n-p+1$ belongs to the algebraic ideal generated by $\rho^{1}, \ldots, \rho^{m-n}$.

The functions $\rho=\left(\rho^{1}, \ldots, \rho^{m-n}\right)$ in Theorem 2.2 are the first integrals of the Pfaffian system (1).

Example 2.3. Let $M=\mathbb{R}^{4}$ and $\theta=\left(\theta^{1}, \theta^{2}\right)$, where

$$
\begin{aligned}
& \theta^{1}=d x^{1}, \\
& \theta^{2}=d x^{2}+x^{3} d x^{4} .
\end{aligned}
$$

Let $\omega^{1}=d x^{3}, \omega^{2}=d x^{4}$. We see that $d \theta^{2}=\omega^{1} \wedge \omega^{2}$, and therefore, $\theta$ is not integrable. By inspection we see that $\rho\left(x^{1}, x^{2}, x^{3}, x^{4}\right):=x^{1}$ satisfies (2.1), therefore, $x^{1}=$ constant gives foliation by reduced submanifolds of dimension 3 .

Example 2.4. Let $M=\mathbb{R}^{4}$ and $\theta=\left(\theta^{1}, \theta^{2}\right)$, where

$$
\begin{aligned}
& \theta^{1}=d x^{1}+x^{2} d x^{3}, \\
& \theta^{2}=d x^{2}+x^{3} d x^{4} .
\end{aligned}
$$

Let $\omega^{1}=d x^{3}, \omega^{2}=d x^{4}$. By a straight forward calculation we see that

$$
\begin{aligned}
& d \theta^{1}=\theta^{2} \wedge \omega^{1}+x^{3} \omega^{1} \wedge \omega^{2}, \\
& d \theta^{2}=\omega^{1} \wedge \omega^{2} .
\end{aligned}
$$

To obtain the largest integrable subsystem is equivalent to finding a nondegenerate function $\rho$ such that

$$
d \rho=f \theta^{1}+g \theta^{2} .
$$

For $j=1,2,3,4$, let $f_{j}, g_{j}$, be defined by

$$
\begin{aligned}
& d f=f_{1} \theta^{1}+f_{2} \theta^{2}+f_{3} \omega^{1}+f_{4} \omega^{2}, \\
& d g=g_{1} \theta^{1}+g_{2} \theta^{2}+g_{3} \omega^{1}+g_{4} \omega^{2} .
\end{aligned}
$$


By applying $d$ to $(2.3)$ we have

$$
\begin{aligned}
& \left(-f_{2}+g_{1}\right) \theta^{1} \wedge \theta^{2}+\left(-f_{3}\right) \theta^{1} \wedge \omega^{1}+\left(-f_{4}\right) \theta^{1} \wedge \omega^{2} \\
& +\left(f-g_{3}\right) \theta^{2} \wedge \omega^{1}+\left(-g_{4}\right) \theta^{2} \wedge \omega^{2}+\left(x^{3} f+g\right) \omega^{1} \wedge \omega^{2}=0 .
\end{aligned}
$$

Setting the coefficients in $(2.5)$ zero we have $f=g=0$, which is absurd. Thus we see that there is no such $\rho$.

\section{References}

[1] R. Bryant, Exterior differential system, Lectures at Duke Univ. noted by Sungho Wang.

[2] R. Bryant, S. S. Chern, R. Gardner, H. Goldschmidt, and P. Griffiths, Exterior Differential Systems, Springer-Verlag, New York, 1991.

[3] A. Clebsch, Über die simultane Integration linearer partieller Differentialgleichungen, J. Reine Angew. Math. (Crelle) 65 (1866), 257-268.

[4] E. A. Coddington and N. Levinson, Theory of Ordinary Differential Equations, McGraw-Hill, New York, NY, 1955.

[5] F. Deahna, Über die Bedingungen der Integrabilitat, J. Reine Angew. Math. 20 (1840), 340-350.

[6] G. Frobenius, Über das Pfaffsche probleme, J. Reine Angew. Math. 82 (1877), 230-315.

[7] P. Griffiths and G. Jensen, Differential systems and isometric embeddings, Annals of Mathematics Studies, 114. The William H. Roever Lectures in Geometry. Princeton University Press, Princeton, NJ, 1987.

[8] C. K. Han, Solvability of overdetermined PDE systems that admit a complete prolongation and some local problems in CR geometry, J. Korean Math. Soc. 40 (2003), no. 4, 695-708.

[9] _ Pfaffian systems of Frobenius type and solvability of generic overdetermined $P D E$ systems, Symmetries and overdetermined systems of partial differential equations, 421-429, IMA Vol. Math. Appl., 144, Springer, New York, 2008.

[10] Generalization of the Frobenius theorem on involutivity, J. Korean Math. Soc. 46 (2009), no. 5, 1087-1103.

[11] C. K. Han and K. H. Lee, Integrable submanifolds in almost complex submanifolds, preprint.

[12] C. K. Han and G. Tomassini, Complex submanifolds in real hypersurfaces, preprint.

[13] M. Kuranishi, On E. Cartan's prolongation theorem of exterior differential systems, Amer. J. Math. 79 (1957), 1-47.

[14] S. Wang, Exterior differential system, 2005 lectures at Seoul Nat. Univ. based on R. Bryant's Duke lectures.

[15] F. Warner, Foundations of Differentiable Manifolds and Lie Groups, Scott, Foresman and Co., Glenview, IL, 1971.

Department of Mathematics

Seoul National University

Seoul 151-742, Korea

E-mail address: ckhan@snu.ac.kr 